\title{
Fatores associados ao uso de substâncias psicoativas em estudantes de uma universidade pública do sul do Maranhão
}

\author{
Factors associated with the use of psychoactive substances in students of a public \\ university in the south of Maranhão
}

\section{Gerson Alves Rodrigues Júnior ${ }^{1}$, Fernanda Oliveira Queiroz ${ }^{\mathbf{1}}$, Hesse do Nascimento Lima ${ }^{\mathbf{1}}$, Leonardo Silva Nóbrega ${ }^{1}$, Iraciane Rodrigues Nascimento Oliveira ${ }^{2}$}

Rodrigues Junior GA, Queiroz FO, Lima HN, Nobrega LS, Oliveira IRN. Fatores associados ao uso de substâncias psicoativas em estudantes de uma universidade pública do sul do Maranhão / Factors associated with the use of psychoactive substances in students of a public university in the south of Maranhão. Rev Med (São Paulo). 2020 maio-jun.;99(3):220-9.

RESUMO: Introdução: O uso de substâncias psicoativas (SPA) pela humanidade é extremamente antigo e seu consumo no decorrer da história possui amplas finalidades. No Brasil, o seu uso fez parte da cultura dos povos antigos na realização de rituais e festas, sendo um fenômeno conhecido desde os tempos da colonização portuguesa. O beber e a utilização de outras drogas fazem parte da cultura brasileira, traduzindo-se em um ato dinâmico que se modifica no intercurso do tempo e espaço. Objetivo: Analisar os fatores associados ao "uso de SPA na vida" dentre universitários no sul do Maranhão. Materiais e métodos: Esse estudo caracteriza-se como quantitativo, analítico e transversal. Para a obtenção da amostra final de 309 alunos, utilizou-se como material de análise o preenchimento de questionário ASSIST, devidamente adaptado para a pesquisa em questão. Após o preenchimento, realizou-se a análise estatística desses questionários através do teste qui quadrado a fim de identificar as associações existentes entre o uso de SPA e cada fator estudado. Resultados: Os fatores associados ao uso de SPA lícitas, em ordem decrescente, foram: ser praticante de alguma religião, possuir pessoa próxima que faz uso de SPA, possuir religião. Já os fatores de maior relevância que levaram ao uso das SPA ilícitas, em ordem decrescente, foram: possuir religião, ser praticante de alguma religião, orientação sexual. Conclusão: Verificou-se que os fatores associados ao uso de SPA são múltiplos e amplos, podendo ser sociais, psicológicos ou mesmo religiosos como, por exemplo, ser ou não praticante de uma religião, dentre outros fatores. Baseado nessa diversidade, e no resultado obtido de que grande parte dos estudantes consideraram não serem capazes de pensar criticamente a respeito das SPA, sugere-se a necessidade de pesquisas complementares, indicando que abordagens mais efetivas a respeito das SPA devem ser desenvolvidas.

Descritores: Drogas ilícitas; Alcoolismo; Tabagismo; Estudantes; Universidades; Fatores de proteção; Fatores de Risco.

\begin{abstract}
Introduction: The use of psychoactive substances (SPA) by humanity is extremely old and its consumption throughout history has broad purposes. In Brazil, its use was part of the culture of the ancient peoples in the performance of rituals and parties, being a known history since the times of Portuguese colonization. How drinking and using other drugs are part of Brazilian culture, translating it into a dynamic act that changes the exchange of time and space. Objective: Analyzing the factors associated with the "use of SPA in life" among university students in Maranhão. Materials and methods: This study describes it as quantitative, analytical and transversal. To test the final sample of 309 students, use the ASSIST questionnaire, suitable for the research in question, as a material for analysis or completion. After completing, a statistical analysis of these questionnaires was performed, using the square test in order to identify the associations between the use of the SPA and each factor studied. Results: The factors associated with the use of SPA, in decreasing order, were: being a practitioner of some religion, having a close person who uses SPA, having religion. The most relevant factors that led to the use of illicit activities in the SPA, in decreasing order, were: having a religion, practicing a religion, sexual orientation. Conclusion: Check if the factors associated with the use of the SPA are multiple and wide, they can be social, psychological or even religious, for example, being or not practicing a religion, among other factors. Based on this diversity, and on the result obtained, most students consider that they are not able to think critically about the use of SPA, it is suggested the need for further research, verifying which more effective approaches about SPA should be developed.
\end{abstract}

Keywords: Illicit drugs; Alcoholism; Tobacco use disorder; Students; Universities; Protective factors; Risk factors.

Instituição: Universidade Federal do Maranhão / UFMA. Centro de Ciências Sociais, Saúde e Tecnologia. Campus II / Imperatriz, MA. Curso de Medicina. 1. Acadêmicos de Medicina da Universidade Federal do Maranhão. ORCID: Rodrigues Junior GA - https://orcid.org/0000-0001-9129-6107, Queiroz FO - https://orcid.org/0000-0003-0048-7229, Lima HN - https://orcid.org/0000-0001-8709-6583, Nobrega LS - https://orcid.org/0000-0002-83977997. Email: gerson.rodriguesjunior@hotmail.com, ffernandaoliveiraqueiroz@gmail.com, hesselima33@gmail.com, leosilvanobrega@gmail.com. 2. Professora, Mestre Titular da Universidade Federal do Maranhão. https://orcid.org/0000-0001-6535-5396. Email: iraciane.rodrigues@gmail.com. Endereço para correspondência: Gerson Alves Rodrigues Júnior. Rua Urbano Santos, 312. Apart. 23. Imperatriz, MA. Email: gerson.rodriguesjunior@, hotmail.com. 


\section{INTRODUÇÃO}

$\mathrm{O}$ uso de substâncias psicoativas (SPA) pela humanidade é uma prática extremamente antiga. Essas substâncias foram e ainda são consumidas com amplas finalidades, podendo-se citar o uso terapêutico, religioso, ostentação e busca pelo prazer como exemplos. Os costumes relacionados as SPA são diversos e possuem peculiaridades conforme o espaço e tempo analisados, sendo o século XX o período em que essa prática foi difundida através de publicidade e marketing, ampliando-se mundialmente o consumo de algumas dessas substâncias ${ }^{1,2}$.

No ano de 2002, em relatório sobre as SPA, a Organização Mundial de Saúde evidenciou que 8,9\% de todas as doenças que assolam a humanidade ocorrem devido ao uso de drogas. Desse montante, o tabaco é responsável por $4,1 \%$ e o álcool por $4,0 \%$, restando $0,8 \%$ a cargo das drogas ilícitas ${ }^{3}$.

Em relação ao Brasil, as SPA faziam parte da cultura dos povos que já habitavam o que viria a tornar-se o país, utilizando-as em rituais e festas. Esse fenômeno é conhecido desde os tempos da colonização portuguesa, tendo-se registros de uma bebida produzida pelos índios através da fermentação da mandioca, denominada "cauim", bem como o uso do tabaco ${ }^{4}$.

O beber e o utilizar de outras SPA fazem parte da cultura e da história brasileira, sendo um ato que não pode, portanto, ser interpretado apenas por si só, necessitandose levar em conta a ocasião em que ocorre, os costumes sociais, locais e culturais, condições psicológicas e físicas do usuário, o contexto e o ambiente em que se faz o uso da droga. Como exemplo cite-se o uso do licor em festas de São João e o vinho no natal ${ }^{4,5}$.

Outro fator importante a ser considerado no Brasil são as publicidades, principalmente de bebidas alcoólicas, que a relacionam a momentos de alegria, muitos amigos, atributos sexuais almejados, dentre outras características desejadas por grande parte da população, e que consequentemente influenciam as diversas camadas da sociedade brasileira, principalmente, o público jovem e adulto ${ }^{4,6}$ ao consumo.

Se, por um lado, o consumo de álcool é incentivado pela mídia, por outro lado, é combatido por leis e programas de saúde. Esse embate, que muitas vezes é desigual, perpetua uma realidade desfavorável aos jovens, e propicia um cenário paradoxal de combate versus o incentivo ao uso de bebidas alcoólicas ${ }^{7,8}$, sendo que a utilização precoce de SPA entre jovens favorece complicações futuras nesses indivíduos ${ }^{3,9}$.

Inclui-se ainda a esse cenário a conjuntura de que em determinados ambientes da sociedade, tal qual os frequentados por jovens e adultos, o consumo de certas SPA é aceito e até mesmo incentivado, resultando em um faixa etária com alta tendência a realizar o uso dessas drogas $^{8}$. Há, inclusive, trabalhos que evidenciam outras variáveis incentivadoras nesses locais, como por exemplo os cartazes das festas universitárias de Uberlândia-MG, onde $93,3 \%$ apresentavam conteúdos relacionados ao uso de bebidas alcoólicas em 2005 e 80,0\% também exibiam as mesmas características em 2007. O estudo concluiu que as propagandas de festas extrapolavam seu papel de promoção de festas, passando a realizar apologia ao consumo de álcool. Percebe-se, desse modo, os universitários como grandes influenciados ${ }^{10}$.

Segundo dados encontrados por diversos autores ${ }^{11-15}$, o uso das SPA, em sua maioria, é consumido em maior quantidade pelo público universitário se comparado com a população em geral ${ }^{16}$. Um dos fatores apontados como causa é que, ao ingressar na universidade, o novo estudante têm poucos recursos comportamentais e pouca experiência ${ }^{17}$, necessitando, muitas vezes, fazer novos amigos e incorporar novos hábitos ${ }^{18,19}$ tal qual fazer o uso de bebidas alcoólicas ${ }^{17,19,20}$. Esse cenário torna esse período da vida acadêmica, principalmente no início, extremamente importante no que se refere ao futuro envolvimento do universitário com o uso de $\mathrm{SPA}^{18,21,22}$.

Esse, portanto, é um grupo de notável interesse, visto que exerce um papel de grande importância no futuro e no desenvolvimento da sociedade brasileira. Tais estudantes, por receberem parte dos investimentos científicos do país, bem como por terem a possibilidade de colaborar com novas formas de agir, com a formação de opiniões e influenciar diversos setores da sociedade, pelas futuras funções que deverão exercer, são indivíduos que demandam um melhor estudo sobre uso de SPA ${ }^{16,23}$.

Evidencia-se, desse modo, a relevância de estudos nessa área, pois o uso de SPA sofre constante variação conforme o local e período em que é analisado, sendo algo dinâmico e com diversas facetas ${ }^{4}$. A maioria dos estudos acerca da prevalência do uso de SPA entre esses discentes concentra-se na região sudeste, principalmente em São Paulo, ou em capitais, tendo poucos dados sobre outras regiões, particularmente no interior do país ${ }^{16,24}$. Logo, necessita-se de mais estudos epidemiológicos para se desenvolver, através de bases científicas, estratégias adequadas as novas formas e fatores que influenciam o usar dessas substâncias nos estudantes de todo o país ${ }^{4,23,25}$. À vista disso, torna-se extremamente relevante e importante o estudo em uma Universidade Federal ao sul do Maranhão, no interior do estado.

O estudo em questão teve como objetivo analisar os fatores associados ao uso de SPA dentre os universitários de uma Universidade Federal no sul do Maranhão.

\section{MATERIAL E MÉTODOS}

O presente estudo caracteriza-se por ser quantitativo, analítico e transversal. Foi realizado em uma universidade federal localizada no sudoeste do Maranhão, no interior 
do estado. Possuindo dois campus, um situado no centro da cidade e outro em um bairro periférico, a universidade polariza alunos de diversos locais do Brasil.

Os cursos escolhidos para se realizar a amostra foram os das áreas da saúde, humanas, sociais e exatas, respectivamente: medicina, jornalismo, direito e engenharia de alimentos. Desses cursos, dois estão no campus do centro da cidade, direito e jornalismo, e dois no campus mais periférico, engenharia de alimentos e medicina. Os períodos de aula desses cursos são: engenharia de alimentos e medicina integrais, jornalismo vespertino e direito noturno.

O levantamento do número de alunos foi realizado pela secretaria da universidade através de seu sistema no mês de fevereiro de 2018, constatando-se 293, 237, 363 e 167 estudantes matriculados em medicina, jornalismo, direito e engenharia de alimentos, respectivamente. $\mathrm{O}$ tamanho da amostra foi calculado com nível de confiança de $95 \%$ e margem de erro de 5\%. Utilizou-se como população ou tamanho do universo a soma do número de acadêmicos dos quatro cursos citados acima, ou seja, 1060 discentes. A amostra obtida foi de 283 universitários.

A critério de proporção, o número de entrevistados nos diferentes cursos foi proporcional à porcentagem que cada curso representa no total da amostra. Desse modo, medicina, jornalismo, direito e engenharia de alimentos representam, respectivamente, $27,6 \% ; 22,4 \%$; $34,2 \%$ e $15,8 \%$ da população total. Convertendo-se essa porcentagem sobre a amostra obtida teve-se 78, 63, 97 e 46 alunos como número mínimo a ser entrevistado de cada curso, seguindo-se a mesma ordem.

A coleta de dados foi realizada de setembro de 2018 até dezembro do mesmo ano. Todos os questionários são do tipo anônimo e de autopreenchimento, sendo feitos presencialmente, após a assinatura do Termo de Consentimento Livre e Esclarecido (TCLE). Reservou-se o TCLE recolhido de forma separada aos questionários, para se garantir e preservar o anonimato dos envolvidos na pesquisa.

Foram respondidos 323 questionários, sendo que apenas 14 deles não foram considerados aptos para análise por terem sido preenchidos indevidamente ou pela falta de preenchimento de todos os campos. Com isso obtevese 309 questionários considerados aptos para realizar a análise, tendo-se um aproveitamento maior de 95\% dos questionários aplicados.

Fluxograma 1. Descrição da amostragem do estudo. Imperatriz, Maranhão, Brasil, 2018, n=309

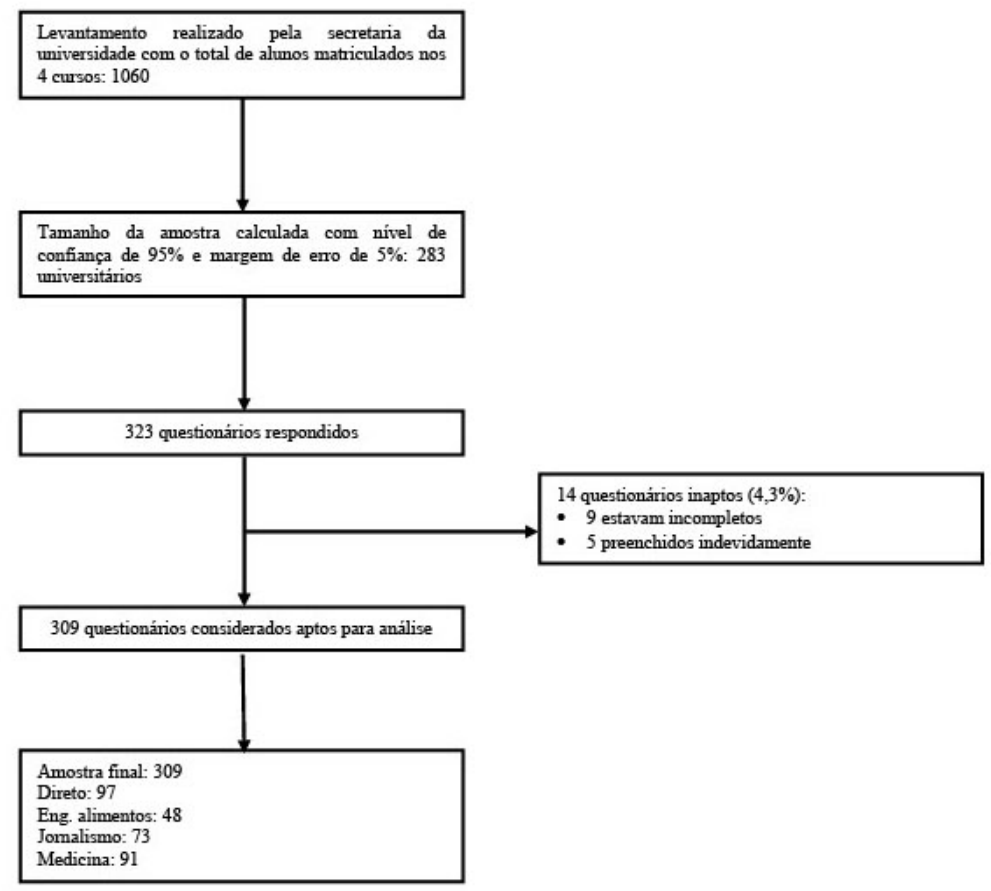

ASSIST, questionário utilizado pela OMS, onde se analisa o consumo de drogas psicoativas na vida e nos últimos três meses. ASSIST vem da sigla Alcohol, Smoking and Substance Involvement Test, uma possível tradução em português seria "Teste para envolvimento com álcool, tabaco e substâncias". Junto a esse questionário também foi utilizado outro, com perguntas abordando os aspectos socioeconômico, cultural e psicológico, bem como 
perguntas sobre o curso feito na universidade e a respeito de como é realizado o uso de SPA pelos universitários.

A pesquisa em questão seguiu as normas da Resolução 466/12 do Conselho Nacional de Saúde. Para tanto foi aprovada pelo Comitê de Ética em Pesquisa da Universidade Federal do Maranhão (UFMA) de São Luiz, através da Plataforma Brasil, sob o parecer $n^{\circ} 2.891 .982$, CAE n ${ }^{\circ}$ 94200618.8.0000.5087.

Realizou-se a análise estatística através do teste qui quadrado utilizando-se o software Statistical Package for the Social Sciences - SPSS, versão 22.0, para estudar as associações entre o uso de SPA e cada variável estudada.

As variáveis estudadas foram: curso matriculado e período em que o aluno estudava em 2018, sexo, orientação sexual, idade, renda, religião, se é praticante da religião, local em que realizou o ensino médio, se mora sozinho, estado civil, problemas econômicos, relacionamento familiar, isolamento social, prática de exercícios, sono satisfatório, sentir-se útil, ver sentido na vida, histórico de suicídio, se faz a faculdade desejada, se é o primeiro curso universitário, sente-se pressionado pela universidade, sente-se pressionado pelos parentes, tempo livre suficiente, se trabalha, se participa de atividades acadêmicas, possui pessoa próxima que faz uso, educação prévia sobre SPA.

\section{RESULTADOS}

O sexo feminino compôs $52,4 \%$ da amostra. A orientação sexual prevalente dentre todos os estudantes foi a heterossexual $(85,4 \%)$. Dos entrevistados: $42,4 \%$ possuem relacionamento estável, faixa etária entre 20 e 24 anos $(53,4 \%)$, moram com os pais ou familiares $(64,1 \%)$, cursaram o ensino médio em escola pública $(61,2 \%)$, têm o sonho de realizar outra faculdade (38,2\%). A renda indicada pelo maior número de alunos foi entre 1 e 3 salários mínimos por pessoa da família $(50,2 \%)$, sendo que desses universitários $80,0 \%$ possuem religião e, dentre esses, apenas 56,3\% são praticantes (Tabela 1 ).

Tabela 1. Religião e a pratica religiosa entre os universitários analisados. Imperatriz, Maranhão, Brasil, 2018, N=309

\begin{tabular}{lcc}
\hline Religião & $\mathbf{n}$ & $\mathbf{\%}$ \\
\hline Católico & 127 & 41,1 \\
Evangélico & 101 & 32,7 \\
Espírita & 10 & 3,2 \\
Outra & 9 & 2,9 \\
Não tem religião & 57 & 18,4 \\
Ateu & 5 & 1,6 \\
Total & 309 & 100 \\
\hline Praticante & $\mathbf{n}$ & $\mathbf{\%}$ \\
\hline Sim & 174 & 56,3 \\
Não & 135 & 43,7 \\
Total & 309 & 100 \\
\hline
\end{tabular}

As prevalências sobre o uso de SPA na vida e nos últimos 3 meses, quando confrontados, demonstraram pouca variação, O que pode apontar um uso crônico dessas substâncias. Já o uso de SPA ilícitas na vida teve índices diminuídos, se comparados com o uso geral das SPA entre os estudantes analisados. Essas prevalências estão descritas nas Tabelas 2 e 3:

Tabela 2. Uso de SPA na vida, nos últimos 3 meses e de SPA ilícitas entre os estudantes por curso matriculado. Imperatriz, Maranhão, Brasil, 2018, N=309

\begin{tabular}{lccccc}
\hline Uso de SPA na vida & Direito & Eng. Alimentos & Jornalismo & Medicina & Total \\
\hline $\mathrm{n}$ & 67 & 28 & 47 & 71 & 213 \\
$\%$ & 69,1 & 58,3 & 64,4 & 78,0 & 68,9 \\
\hline Nos últimos 3 meses & & & & \\
\hline $\mathrm{n}$ & 59 & 25 & 39 & 67 & 190 \\
$\%$ & 60,8 & 52,1 & 53,4 & 73,6 & 61,5 \\
\hline Uso de SPA Ilícitas na vida & & & & 31 & 83 \\
\hline $\mathrm{n}$ & 17 & 8 & 27 & 34,1 & 26,9 \\
\hline$\%$ & 17,5 & 16,7 & 37,0 & & \\
\hline $\mathrm{n}^{0}$ total de alunos por curso & & & & 91 & 309 \\
\hline $\mathrm{n}$ & 97 & 48 & 73 &
\end{tabular}


Tabela 3. SPA utilizadas pelos universitários. Imperatriz, Maranhão, Brasil, 2018, N=309

\begin{tabular}{lcccc}
\hline \multirow{2}{*}{ SPA usada } & \multicolumn{2}{c}{ Na vida } & \multicolumn{2}{c}{ Nos últimos 3 meses } \\
\cline { 2 - 5 } & $\mathrm{n}$ & $\%$ & $\mathrm{n}$ & $\%$ \\
\hline Derivados do tabaco & 80 & 37,6 & 75 & 39,5 \\
Bebidas alcoólicas & 208 & 97,7 & 183 & 96,3 \\
Maconha & 65 & 30,5 & 64 & 33,7 \\
Cocaína/ Crack & 10 & 4,7 & 10 & 5,3 \\
Anfetaminas ou êxtase & 14 & 6,6 & 14 & 7,4 \\
Inalantes & 22 & 10,3 & 22 & 11,6 \\
Hipnóticos/sedativos & 17 & 8,0 & 17 & 8,9 \\
Alucinógenos & 16 & 7,5 & 16 & 8,4 \\
Opióides/opiáceos & 9 & 4,2 & 8 & 4,2 \\
Total & 213 & 100,0 & 190 & 100,0 \\
\hline
\end{tabular}

Os motivos alegados para fazer o uso de SPA na vida pelos estudantes, em ordem decrescente, foram: não sei opinar $(39,9 \%)$, diversão $(32,4 \%)$, alívio do estresse $(20,7 \%)$, socializar $(16,9 \%)$, curiosidade $(11,7)$, busca por prazer $(9,4 \%)$, para sentir-se melhor $(8,9 \%)$ e fuga dos problemas $(8,0 \%)$.

Tabela 4. Fatores associados ao uso de SPA na vida entre os universitários. Imperatriz, Maranhão, Brasil, 2018, N=309

\begin{tabular}{lc}
\hline Variável & p-valor \\
\hline Ser praticante de alguma religião & 0,0000027 \\
Possuir uma pessoa próxima que faz uso de SPA & 0,0000034 \\
Possuir religião* & 0,000070 \\
Não morar com parentes ou familiares & 0,0033 \\
Orientação sexual & 0,0052 \\
Acreditar que a faculdade favorece o uso de & 0,012 \\
SPA & 0,037 \\
Local onde cursou o ensino médio & 0,046 \\
Possuir um bom relacionamento familiar & p-valor de significância $(<0,05) ;{ }^{*}$ Apenas para a religião evangélica
\end{tabular}

Tabela 5. Fatores associados ao uso de SPA ilícitas na vida entre os universitários. Imperatriz, Maranhão, Brasil, 2018, N=309

\begin{tabular}{lc}
\hline Variável & p-valor \\
\hline Possuir religião* & 0,000033 \\
Ser praticante de alguma religião & 0,00038 \\
Orientação sexual & 0,00083 \\
Não morar com parentes ou familiares & 0,0011 \\
Possuir um bom relacionamento familiar & 0,0016 \\
Curso matriculado & 0,0045 \\
Ter problemas econômicos & 0,0077 \\
Sentir-se pressionado por familiares & 0,0081 \\
Possuir uma pessoa próxima que faz uso de SPA & 0,0086 \\
Histórico pessoal de suicídio & 0,026 \\
Sentir-se útil & 0,033 \\
\hline
\end{tabular}

p-valor de significância $(<0,05)$; ${ }^{*}$ Católica e evangélica
Ademais, 38,2\% dos estudantes responderam que tem vontade de realizar outro curso e $68,3 \%$ dos alunos entrevistados acreditam que a universidade favorece o uso de SPA. Ao serem questionados se receberam algum tipo de educação em relação ao uso de SPA no ensino fundamental ou médio, 78,0\% assinalou que sim, entretanto apenas $11,0 \%$ afirmou que a educação atual do Brasil ensina a pensar criticamente sobre o uso de SPA.

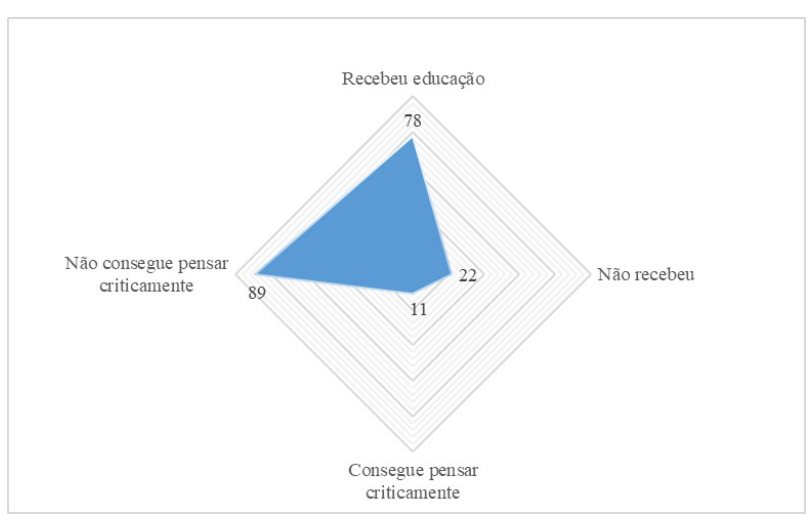

Figura 1. Porcentagem dos universitários que receberam algum tipo de educação sobre o uso de SPA no ensino fundamental e/ ou médio versus a opinião dos próprios alunos a respeito da capacidade de pensar criticamente em relação ao uso das SPA. Imperatriz, Maranhão, Brasil, 2018, N=309

Os responsáveis por fornecer a educação sobre o uso de SPA foram, em ordem decrescente: $28,2 \%$ professores do ensino fundamental ou médio, $21,6 \%$ profissionais da área de saúde, $17,0 \%$ não declararam, $14,9 \%$ por diversos profissionais, $13,3 \%$ por policiais ou pelo PROERD e 5,0\% pelos parentes. Houve significância de maior uso de SPA na vida (p-valor 0,024$)$ quando a educação foi fornecida por professores, profissionais da área da saúde, profissionais diversos e policiais ou PROERD em relação aos que não declararam o tipo de profissional que ministrou as aulas a respeito do consumo de drogas. 


\section{DISCUSSÃO}

Ressalta-se nos novos estudos em relação as SPA a relevância de se analisar os diversos fatores associados a essa prática, não se pautando apenas nos efeitos das SPA no SNC, tal qual antigamente, quando não se levava em conta o contexto em que se realizavam as pesquisas ${ }^{26}$, pois as recentes análises apontam para a soma de ações combinadas e complexas de fatores sociais, neurobiológicos e psicológicos como causadores de problemas e dependência ao usuário ${ }^{3}$.

$\mathrm{O}$ uso de questionários para colher informações sobre o uso de drogas, apesar de muitas vezes questionados, ainda é o método mais utilizado e mostra-se eficaz, pois tem baixo custo, assegura o anonimato, tem pouca rejeição e são vistos pelos estudantes como uma forma de prevenção e não repressão, tendo, portanto, grande confiabilidade em seus resultados ${ }^{27}$.

Devido ao grande número de variáveis possíveis optou-se por utilizar o "uso na vida" das SPA para análise dos fatores associados ao uso de SPA entre os universitários. Seguindo, desse modo, o mesmo padrão adotado pelo CEBRID, órgão que foi responsável por pesquisas a nível nacional sobre o uso de SPA. Além disso, esse tipo de uso permite mensurar a presença das SPA de forma ampliada nessa parcela da sociedade ${ }^{1,27}$.

A prevalência do "uso na vida" das drogas lícitas, bebidas alcoólicas e tabaco, encontrada foi de $67,3 \%$ e $25,9 \%$, respectivamente. Os valores do uso de álcool são considerados reduzidos em comparação com outros levantamentos entre universitários, onde essa prevalência ficou acima de $80 \% \%^{1,5,12,13,18,24,25,27-30}$, inclusive abaixo do "uso na vida" em estudo realizado em 2010 na população em geral e universitários brasileiros $(74,6$ e $86,2 \%)^{1}$. Em relação ao uso dos derivados do tabaco encontrou-se também números baixos se comparados a outras universidades, com porcentagens superiores a $30 \%{ }^{5,12,13,18,25,27,28}$, sendo também inferior ao uso da população em geral e universitários brasileiros (44,6 e $46,7 \%$ ). Porém, também há valores similares encontrados em outras pesquisas, $27 \% 0^{30}$ e $22,3 \%{ }^{29}$.

O "uso na vida" de substâncias ilícitas foi de $26,9 \%$, dado semelhante a outras pesquisas realizadas ${ }^{5,25,28}$. Embora a maconha tenha sido a SPA ilícita mais utilizada, o que foi igualmente relatado em outras análises ${ }^{5,18,25}$, a maioria dos estudos indicam os solventes como a SPA ilegal mais usada entre os estudantes de nível superior ${ }^{12,13,24,27,30}$. Os usos variam entre $9,4-17,0 \%$ para maconha e $11,9 \%-46,2 \%$ para solventes. Os números dos alunos analisados no corrente trabalho indicam maior uso na vida quando comparados à população em geral $(22,8 \%)$ e menor se contrapostos com os universitários brasileiros $(48,7 \%)^{1}$.

Os resultados acerca do "uso na vida" de substâncias legais sugerem a universidade em si como fator não influenciador para o consumo de SPA, corroborando com outras análises ${ }^{12,27}$ comparadas. Porém os dados das SPA ilícitas indicam certa influência da faculdade no uso de drogas, assim como outros levantamentos ${ }^{10,24,25,29}$. As investigações existentes em relação à universidade como influenciadora ou não ao uso de drogas são divergentes ${ }^{27}$.

Em relação ao sexo, o sexo masculino teve maior prevalência de uso de SPA com $72,1 \%$, contra $66,2 \%$ do sexo feminino, porém sem diferença com p-valor significante $(0,25)$, valor muito próximo ao encontrado por Lucas et al. ${ }^{27}$. Houve p-valor de relevância para o consumo de alucinógenos $(0,024)$, êxtase $(0,017)$ e tabaco $(0,020)$ no sexo masculino. Na publicação de Lemos et al. ${ }^{24}$ também houve uso de tabaco significativamente maior no sexo masculino. Registrou-se maior uso de sedativos no sexo feminino $(7,4 \%$ contra $3,4 \%)$, porém não houve associação significante $(0,123)$, analogamente ao relatado pela pesquisa de Stempliuk ${ }^{5}$. Diferente de outras pesquisas que tiveram o uso de êxtase com maior prevalência entre o sexo feminino $0^{5,27}$.

O maior uso no sexo masculino na maioria das drogas pode ser explicado por diversos fatores, dentre eles culturais, a exemplo do apontado por Rios e Dázio et al..$^{9,31}$. Já o uso diminuído de certas substâncias entre as mulheres, a exemplo do álcool, pode ser pela visão social de que a atitude do uso de certas SPA é visto tal qual a algo viril ${ }^{1,31}$.

A ordem decrescente apurada no corrente trabalho, foi semelhante à ordem encontrada na faculdade de medicina da Bahia: Diversão, relaxar e estresse ${ }^{24}$. O estresse também foi apontado como um dos principais motivos em uma universidade de Curitiba $^{28}$. Chama atenção ainda o estudo feito por Kerr-Corrêa et al. ${ }^{13}$, onde $60 \%$ dos entrevistados não soube explicar o motivo do uso, número próximo ao encontrado na presente pesquisa.

A principal causa de uso ter sido "não sei opinar", situação apontada no atual estudo e em estudos anteriores, levanta a hipótese de que uma parcela dos universitários usa drogas sem refletir criticamente sobre elas.

Acerca dos fatores associados ao "uso na vida" de SPA destacam-se os seguintes estudos com resultados similares:

- Ser praticante de alguma religião foi fator protetor para Silva et al. ${ }^{11}$, contrariamente à pesquisa realizada por Andrade et al. ${ }^{32}$ em estudantes do ensino médio;

- Possuir uma pessoa próxima que faz uso de SPA foi documentado como fator de risco em diversos trabalhos, e se aplica tanto para membros da família, quanto para amigos e colegas próximos ${ }^{27,28,33}$;

- A religião mostrou-se como fator protetor para evangélicos de forma similar ao trabalho de Silva et al. ${ }^{11}$, bem como foi documentada a maior incidência de uso de álcool em católicos, no mesmo estudo. Já Funai e Pillon ${ }^{34}$ encontraram maior uso nos universitários que se declaram espíritas, corroborando com o observado no presente trabalho; 
- Igualmente à análise de Magliari et al. ${ }^{35}$, o fator "não morar com parentes ou familiares" mostrou-se como de risco para o uso de SPA "na vida". Outro estudo em que se levantou a porcentagem do tipo de moradia e o uso de SPA entre os universitários foi o de Fiorini e Alves ${ }^{12} \mathrm{e}$, ainda que não se tenha feito a análise da associação entre as duas variáveis, foi constatado uma taxa alta de "uso na vida" de SPA (88\%). Além disso, somente 19\% desses entrevistados afirmaram morar com pais/familiares;

- A orientação sexual não foi relatada em outros estudos, porém no presente trabalho, declarar-se homossexual ou bissexual foi um fator de risco para o uso de SPA, diferentemente dos autodeclarados heterossexuais (p-valor 0,0052);

- O local onde se cursou o ensino médio, ou seja, o tipo de escola frequentada pelos estudantes nesse período apresentou-se como fator de risco (p-valor 0,037), inclusive no estudo feito por Stronski et al. ${ }^{36}$, atribuindo-se maior uso nos aluno de escolas privadas;

- Não possuir um bom relacionamento familiar foi considerado fator de risco (p-valor 0,046), o que também foi relatado nas pesquisas de Fischer e Vansan, bem como Stronki et al ${ }^{36,37}$, quando relatado pelo estudante o sentimento de que seus familiares o desvalorizam. Além disso foi indicado que a qualidade das relações familiares tem impacto quanto ao uso de SPA entre estudantes;

- O uso de SPA na vida não teve relação com a quantidade de exercícios realizados na semana ( $\mathrm{p}$-valor 0,074), assim como relatado por Magliari et al. ${ }^{35}$. Entretanto, segundo o estudo de Stronski et al. ${ }^{36}$, frequentar clubes esportivos com regularidade foi fator protetor para o uso de drogas ilícitas, com exceção da maconha. Já nos estudos apresentados por Silva et al. ${ }^{11}$, os resultados demonstram o contrário, apontando que a maior frequência nas associações esportivas é observada em usuários de drogas lícitas e ilícitas;

- Ter tempo livre suficiente não foi considerado fator de risco para o uso de SPA (p-valor 0,72), assim como ser do sexo masculino (p-valor 0,25 ), contrariando os resultados encontrados por Kerr-Corrêa et al..$^{13} \mathrm{e}$ Silva et al. ${ }^{11,13}$, anteriormente;

- Não houve relação entre a renda familiar alta e o uso de SPA (p-valor 0,25), distintamente do apontado na investigação realizado por Silva et al. ${ }^{11}$. A disparidade entre os resultados pode ser em decorrência do fato de alguns alunos não se sentirem bem em escrever sobre sua renda ou de sua família, como relatado similarmente por Kerr-Corrêa et al. ${ }^{13}$.

Ademais, destaca-se a repetição da maioria dos fatores presentes tanto no "uso na vida" de SPA lícitas como das ilícitas, sugerindo, dessa forma, que os mesmos fatores podem ter implicações no uso de substâncias diferentes. Evidencia-se ainda, no uso de substâncias ilegais, a associação com o histórico de tentativa de suicídio (p-valor 0,026), tal qual relatado por Ficher e $\operatorname{Vansan}^{37} \mathrm{e}$ por Moreira et al..$^{38}$.

Existem diversos estudos a respeito do uso de SPA, porém a maioria concentra-se nos cursos das áreas biológicas/saúde e, principalmente, no curso de medicina. São poucas as publicações que representam toda a população universitária e somente alguns levantamentos permitem a comparação entre os diferentes cursos existentes. Sendo assim, vislumbra-se a importância de se terem novas pesquisas em relação ao uso de SPA envolvendo estudantes universitários de cursos diversos, a fim de que sejam representados de forma fidedigna os fatores associados e o uso nessa população como um todo ${ }^{25}$.

Realça-se ainda outros pontos notáveis encontrados:

- Mesmo que não seja o objetivo do trabalho, ao confrontar-se o uso de SPA na vida e o uso nos últimos 3 meses de tabaco e de SPA ilícitas, observam-se números muito semelhantes, insinuando um provável uso crônico dessas substâncias;

- O curso de jornalismo foi o único a ter maconha como segunda substância mais utilizada $(51,1 \%)$, superando o tabaco. Esse resultado pode estar atrelado à divergências entre as políticas de desestímulo do fumo de cigarros de tabaco, decorrentes do Programa Nacional de Controle ao Tabagismo, realizado pelo INCA, desde $1986^{39}$ e as atuais políticas mundiais que seguem na direção da regularização e legalização da maconha ${ }^{40}$ (que podem ser interpretadas como um incentivo ao uso dessa SPA em detrimento do uso do tabaco).

Referentemente ao curso de medicina, foi verificado que os fatores "uso na vida" (p-valor 0,037 ), uso nos "últimos três meses" (p-valor 0,040) e "uso de SPA ilícitas na vida" $(0,030)$ foram os únicos significativos e demonstraram possuir íntima relação com os períodos cursados. Os demais fatores, verificados nos outros cursos, não foram relevantes para o uso de substâncias por esses estudantes.

Esse padrão verificado foi muito semelhante ao relatado por outras pesquisas ${ }^{13,35,41}$, que igualmente demonstraram que o uso de drogas na medicina é tanto maior quanto mais avançados forem os períodos, com a ressalva de que no presente estudo observou-se um uso acima do esperado no $1^{\circ}$ período e nos períodos mais avançados, exceto no $10^{\circ}$.

$\mathrm{Na}$ análise referente ao uso de drogas ilícitas, ainda por estudantes de medicina, também foi verificado no corrente trabalho o aumento do consumo conforme o decorrer do curso, principalmente com relação aos opióides, sedativos e alucinógenos, o que foi igualmente relatado por Kerr-Corrêa et al. ${ }^{11,13}$ e Silva et al. ${ }^{11,13}$, referentemente aos sedativos. Tem-se como possível explicação desse fenômeno o fato de que esses alunos, por estarem no final do curso, sentem-se mais pressionados, têm seu ritmo de sono constantemente modificado e possuem fácil acesso a medicamentos estando dentro do hospital.

Com base no apresentado, sugere-se o curso de 
medicina como possível influenciador ao uso de SPA. O presente trabalho corrobora com tese de estudo anterior ${ }^{31}$ que apresenta como hipótese de fator de favorecimento o maior contato com o sofrimento alheio, comum a esses estudantes. Ressalte-se que esses alunos teoricamente possuem maior conhecimento a respeito do uso e efeito das SPA e, mesmo assim, consomem mais que os outros cursos, o que se traduz em um comportamento paradoxal.

Salienta-se, por fim, a importância do que traduzem os dados relacionados à educação fornecida por diversos profissionais, de diversas áreas do conhecimento e que acabam por se revelar em métodos não efetivos para o desenvolvimento do pensamento crítico a respeito do uso de SPA, conforme evidenciado pela maioria dos entrevistados.

Por conseguinte, levanta-se a crítica de que a formação em relação às SPA é deficiente, tanto aquelas vindas de profissionais que ministram as aulas quanto da educação primária, secundária e terciária brasileira. Conjuntura ainda mais preocupante se infere à situação dos cursos da área da saúde, vez que muitos desses profissionais, futuramente, irão lidar com o uso de SPA em seus pacientes. Como final recomendação, sugere-se a realização de mudanças nas matrizes universitárias com implementação de políticas mais eficazes de orientação sobre o uso de SPA, sendo que o aperfeiçoamento da capacidade de elaboração de pensamentos críticos em seus alunos é premente e pode ser alcançado também através de informações científicas e aulas, assim como proposto por outros pesquisadores ${ }^{6,8,13}$.

\section{CONCLUSÕES}

Os principais fatores associados ao uso de SPA entre os estudantes, como ser praticante de alguma religião, possuir uma pessoa próxima que faz uso de SPA, orientação sexual, não morar com parentes ou familiares, curso matriculado, dentre outros, demonstraram a relevância de diversas áreas, desde culturais, familiares, religiosas até sociais e educacionais, como influenciadoras desse consumo, apresentando uma conjuntura vasta de múltiplos fatores, tanto protetivos quanto de risco.

Devido a essa multifatoriedade e complexibilidade, entendeu-se que há a necessidade de novas pesquisas e análises que caminhem para o aperfeiçoamento dos estudos a esse respeito, aprimorando a capacidade de atuação e identificação desses fatores, a fim de que se aperfeiçoem as ferramentas que possam estimular o pensamento crítico, vez que as atuais políticas demonstraram, nesse aspecto, não serem totalmente eficazes, sob o ponto de vista dos entrevistados.

Importante ainda se destacar a necessidade de mudança da educação brasileira sobre o uso de SPA, pois, unicamente através da educação com uma formação baseada na ciência e no desenvolvimento de senso crítico, é possível refletir e entender a realidade, para, desse modo, atuar de forma ativa em sua transformação.

Agradecimentos: À minha família, meus professores e amigos, à minha irmã, Andressa, e à minha esposa e eterna namorada, Iara.

Participação dos Autores: Rodrigues Junior GA: pesquisa bibliográfica, coleta de dados, análise de dados, discussão dos dados, elaboração do manuscrito, revisão bibliográfica. Lima HN: coleta de dados, tabulação de dados, análise de dados e discussão dos dados, atualização das referências. Nobrega LS: coleta de dados, tabulação de dados, análise de dados e discussão dos dados, atualização das referências. Queiroz FO: coleta de dados, discussão dos dados, auxílio na elaboração do manuscrito, atualização das referências. Oliveira $I R N$ : orientadora do trabalho, participando de todas etapas, exceto coleta e tabulação de dados.

\section{REFERÊNCIAS}

1. CEBRID - Centro Brasileiro de Informações sobre Drogas Psicotrópicas Universidade Federal de São Paulo, Departamento de Psicobiologia, Secretaria Nacional de Políticas sobre Drogas - SENAD. Livreto informativo sobre drogas psicotrópicas. $5^{\mathrm{a}}$ ed. Brasília: CEBRID/SENAD; 2011. Disponível em: https://www.justica.gov.br/central-deconteudo/politicas-sobre-drogas/cartilhas-politicas-sobredrogas/drogaspsicotropicas.pdf.

2. Trindade BPDA, Diniz AV, Sá-Júnior AR. Uso de drogas entre estudantes universitários: uma perspectiva nacional. Rev Med Saúde (Brasília). 2018;7(1):52-60. Disponível em: https://portalrevistas.ucb.br/index.php/rmsbr/article/ view/8641/5721.

3. Organização Mundial da Saúde. Neurociencias: consumo e dependência de substâncias psicoativas. Genebra: Organização Mundial da Saúde; 2004. Disponível em: https:/www.who.int/substance_abuse/publications/en/ Neuroscience_P.pdf?ua=1.

4. Brasil. Ministério da Saúde. O uso de substâncias psicoativas no Brasil. Secretaria Nacional de Políticas sobre Drogas. SUPERA: Sistema para detecção do uso abusivo e dependência de substâncias psicoativas: encaminhamento, intervenção breve, reinserção social e acompanhamento. 9a. ed. Brasília: Ministério da Justiça; 2016.

5. Stempliuk VA. Uso de drogas entre alunos da Universidade de São Paulo: 1996 versus 2001 [tese]. São Paulo: Universidade de São Paulo; 2004. Disponível em: http://www.teses.usp. br/teses/disponiveis/5/5160/tde-24012007-182400/publico/ vladimirpdf.pdf.

6. Porto AO, Rios MA, Souza DA. Influência da mídia televisiva no consumo de bebidas alcoólicas por universitários. SMAD Rev Eletrônica Saúde Mental Alcool Drog. 2018;14(1):52-61. Disponível em: 10.11606/issn.1806-6976.smad.2018.000394

7. Pechansky F, Szobot CM, Scivoletto S. Uso de álcool entre adolescentes: conceitos, características epidemiológicas e fatores etiopatogênicos. Rev Bras Psiquiatr. 2004;26(suppl 1):14-7. http://dx.doi.org/10.1590/S151644462004000500005 .

8. Camargo ECP, Gonçalves JS, Felipe AOB, Fava SMCL, Zago MMF, Dázio EMR. Uso e abuso de drogas entre 
universitários e a sua interface com as políticas públicas. SMAD Rev Eletrônica Saúde Ment Álcool Drog (Edição em Port.). 2019;15(4):1-9. doi: 10.11606/issn.1806-6976. smad.2019.000364.

9. Dázio EMR, Zago MMF, Fava SMCL. Use of alcohol and other drugs among male university students and its meanings. Rev Esc Enferm USP..2016;50(5):785-91. doi: http://dx.doi. org/10.1590/S0080-623420160000600011.

10. Musse AB. Apologia ao uso e abuso de álcool entre universitários: uma análise de cartazes de propaganda de festas universitárias. SMAD Rev Eletrônica Saúde Mental Alcool Drog. 2008;4(1):1-8. https://doi.org/10.11606/ issn.1806-6976.v4ilp01-13.

11. Silva LVER, Malbergier A, Stempliuk VA, Andrade AG. Fatores associados ao consumo de álcool e drogas entre estudantes universitários. Rev Saude Publica. 2006;40(2):2808. https://doi.org/10.1590/S0034-89102006000200014.

12. Fiorini JE, Alves AL, Ferreira LR, Fiorini CM, Durães SW, Santos RLD, et al. Use of licit and illicit drugs at the University of Alfenas. Rev Hosp Clin Fac Med Sao Paulo. 2003;58(4):199-206. https://doi.org/10.1590/S004187812003000400003 .

13. Kerr-Corrêa F, Andrade AG, Bassit AZ, Boccuto NMVF. Uso de álcool e drogas por estudantes de medicina da Unesp. Rev Bras Psiquiatr. 1999;21(2):95-100. https://doi.org/10.1590/ S1516-44461999000200005.

14. Ham LS, Hope DA. College students and problematic drinking: a review of the literature. Clin Psychol Rev. 2003;23(5):719-59. doi: 10.1016/s0272-7358(03)00071-0.

15. Jia Z, Jin Y, Zhang L, Wang Z, Lu Z. Prevalence of drug use among students in mainland China: a systematic review and meta-analysis for 2003-2013. Drug Alcohol Depend. 2018;186:201-6. https://doi.org/10.1016/j. drugalcdep.2017.12.047.

16. Brasil. Secretaria Nacional de Políticas sobre Drogas, Arthur Guerra de Andrade; Paulina do Carmo Arruda Vieira Duarte; Lúcio GA, organizadores. I Levantamento Nacional sobre o Uso de Álcool, Tabaco e Outras Drogas entre Universitários das 27 Capitais Brasileiras. Brasília: GREA/IPQ-HC/FMUSP; 2010.

17. Wood MD, Read JP, Palfai TP, Stevenson JF. Social influence processes and college student drinking: the mediational role of alcohol outcome expectancies. J Stud Alcohol. 2001;62(1):3243. http://www.jsad.com/doi/10.15288/jsa.2001.62.32.

18. Pillon SC, O'Brien B, Chavez KAP. The relationship between drugs use and risk behaviors in brazilian university students. Rev Lat Am Enfermagem. 2005;13(spe2):1169-76. https:// doi.org/10.1590/S0104-11692005000800011.

19. Garrido-González I, Bugarín-González R, MachínFernández AJ. Consumo de drogas en estudiantes de enfermería. Enfermería Clín. 2016;26(3):174-80. http:// dx.doi.org/10.1016/j.enfcli.2015.11.006.

20. Skidmore CR, Kaufman EA, Crowell SE. Substance use among college students. Child Adolesc Psychiatr Clin N Am. 2016;25(4):735-53. http://dx.doi.org/10.1016/j. chc.2016.06.004

21. Peuker AC, Fogaça J, Bizarro L. Expectativas e beber problemático entre universitários. Psicol Teor Pesqui. 2006;22(2):193-200. Disponível em: https://www.lume. ufrgs.br/bitstream/handle/10183/20241/000559539. pdf? sequence $=1$.

22. Jalilian F, Ataee M, Matin BK, Ahmadpanah M, Jouybari TA, Eslami AA, et al. Cognitive Factors Related to Drug Abuse Among a Sample of Iranian Male Medical College Students. Glob J Health Sci. 2015;7(5):143-51. doi: 10.5539/gjhs. v7n5p 143 .

23. Fernandes TF, Monteiro BMM, Silva JBM, Oliveira KM, Viana NAO, Gama CAP, et al. Uso de substâncias psicoativas entre universitários brasileiros: perfil epidemiológico, contextos de uso e limitações metodológicas dos estudos. Cad Saúde Coletiva. 2017;25(4):498-507. https://doi. org/10.1590/1414-462x201700040181.

24. Lemos KM, Neves NMBC, Kuwano AY, Tedesqui G, Bitencourt AGV, Neves FBCS, et al. Uso de substâncias psicoativas entre estudantes de medicina de Salvador (BA). Arq Psiquiatr Clin. 2007;34(3):118-24. http://dx.doi. org/10.1590/S0101-60832007000300003.

25. Wagner GA, Andrade AG. Uso de álcool, tabaco e outras drogas entre estudantes universitários brasileiros. Arq Psiquiatr Clin. 2008;35(1):48-54. http://dx.doi.org/10.1590/ S0101-60832008000700011.

26. Hart C. Um preço muito alto: a jornada de um neurocientista que desafia nossa visão sobre as drogas. Rio de Janeiro: Zahar; 2014.

27. Lucas ACS, Parente RCP, Picanço NS, Conceição DA, Costa $\mathrm{KRC}$, Magalhães IRS, et al. Uso de psicotrópicos entre universitários da área da saúde da Universidade Federal do Amazonas, Brasil. Cad Saude Publica. 2006;22(3):663-71. http://dx.doi.org/10.1590/S0102-311X2006000300021.

28. Chiapetti N, Serbena CA. Uso de álcool, tabaco e drogas por estudantes da área de saúde de uma Universidade de Curitiba. Psicol Reflexão Crítica. 2007;20(2):303-13. http://dx.doi. org/10.1590/S0102-79722007000200017

29. Mardegan PS, Souza RS, Buaiz V, Siqueira MM. Uso de substâncias psicoativas entre estudantes de enfermagem. J Bras Psiquiatr. 2007;56(4):260-6. http://dx.doi.org/10.1590/ S0047-20852007000400004.

30. Teixeira RF, Souza RS, Buaiz V, Siqueira MM. Uso de substâncias psicoativas entre estudantes de odontologia da Universidade Federal do Espírito Santo. Cien Saude Colet. 2010;15(3):655-62. http://dx.doi.org/10.1590/S141381232010000300007 .

31. Rios PAA, Matos AM, Fernandes MH, Barbosa AR. Consumo e uso abusivo de bebidas alcoólicas em estudantes universitários do município de Jequié/BA. Rev Saúde Com. 2008;4(2):105-16. Disponível em: http://periodicos2.uesb.br/ index.php/rsc/article/view/129.

32. Andrade ME, Santos IHF, Souza AAM, Silva ACS, Leite TDS, Oliveira CCC, et al. Experimentation with psychoactive substances by public school students. Rev Saude Publica. 2017;51:82. https://doi.org/10.11606/S15188787.2017051006929

33. Lichtenfeld M, Kayson WA. Factors in college students' drinking. Psychol Rep. 1994;74(3):927-30. https://doi. org/10.2466/pr0.1994.74.3.927. 
Rodrigues Junior GA, ET AL. Fatores associados ao uso de substâncias psicoativas em estudantes.

34. Funai A, Pillon SC. Uso de bebidas alcoólicas e aspectos religiosos em estudantes de enfermagem. Rev Eletrônica Enferm. 2011;13(1):24-9. http://dx.doi.org/10.5216/ree. v13i1.8729.

35. Magliari RT, Pagliusi AL, Previero BM, Menezes FR, Feldman A, Novo NF. Prevalência de tabagismo em estudantes de faculdade de medicina. Rev Med. 2008 ;87(4):264. doi: 10.11606/issn.1679-9836.v87i4p264-271.

36. Stronski SM, Ireland M, Michaud P-A, Narring F, Resnick MD. Protective correlates of stages in adolescent substance use: a swiss national study. J Adolesc Heal. 2000;26(6):420-7. doi: 10.1016/s1054-139x(99)00057-9.

37. Ficher AMFT, Vansan GA. Tentativas de suicídio em jovens: aspectos epidemiológicos dos casos atendidos no setor de urgências psiquiátricas de um hospital geral universitário entre 1988 e 2004. Estud Psicol (Campinas). 2008;25(3):361-74. https://doi.org/10.1590/S0103-166X2008000300005.

38. Moreira RMM, Félix TA, Flôr SMC, Oliveira EN, Albuquerque JHM. Análise epidemiológica dos óbitos por suicídio. SANARE (Sobral). 2017;16(1):29-34.
Disponível em: https://sanare.emnuvens.com.br/sanare/ article/viewFile/1136/621.

39. Campos PCM, Gomide M. O Programa Nacional de Controle do Tabagismo (PNCT) na perspectiva social: a análise de redes, capital e apoio social. Cad Saúde Coletiva. 2015;23(4):43644. https://doi.org/10.1590/1414-462X201500040241.

40. Elias C, Oliveira A, Rosa HCM, Wisniewski M. Maconha: história e reflexão diante das problemáticas atuais. In: XV Jornada Científica dos Campos Gerais, 2017. Disponível em: https://www.iessa.edu.br/revista/index.php/jornada/article/ view/347/132.

41. Gomes LS, Barroso CRD, Silvestre VA, Baylão ACDP, Garcia SCM, Pacheco SJB. Consumo de álcool entre estudantes de medicina do Sul Fluminense - RJ. Rev Med. 2018;97(3):260-6. doi: http://dx.doi.org/10.11606/issn.16799836.v97i3p260-266.

Recebido: 24/11/19

Aceito: 27.04 .2020 